second series is identically zero, so the inversion formula does not give an actual solution. Under these circumstances we are forced to leave the question of the completeness of $S_{1}+1$ in $C[0,1]$ unanswered.*

Yale University and Massachusetts Institute of Technology

\title{
GROUPS OF MOTIONS IN CONFORMALLY FLAT SPACES
}

\section{BY JACK LEVINE}

1. Introduction. In this paper we consider the problem of determining the conditions which a conformally flat space must satisfy in order that it may admit a group of motions. These conditions are expressed in Theorem 1. Conformally flat spaces admitting simply transitive groups of motions are considered in the last section. All summations are from 1 through $n$ unless otherwise indicated.

2. Killing's Equations. The equations for determining the possible existence of groups of motions in a metric space are known as Killing's equations and are given by $\dagger$

$$
\xi^{k} \frac{\partial g_{i j}}{\partial x^{k}}+g_{i k} \frac{\partial \xi^{k}}{\partial x^{j}}+g_{j k} \frac{\partial \xi^{k}}{\partial x^{i}}=0 .
$$

If $V_{n}$ is conformally flat, there exists a coordinate system in which $g_{i j}=e_{i} \delta_{j}^{i} h^{2}$, where $e_{i}= \pm 1$. In this coordinate system (1) reduce to

$$
\begin{aligned}
e_{i} \frac{\partial \xi^{i}}{\partial x^{j}}+e_{j} \frac{\partial \xi^{j}}{\partial x^{i}} & =0, \quad(i \neq j, i, j \text { not summed }), \\
\xi^{k} \frac{\partial H}{\partial x^{k}}+\frac{\partial \xi^{i}}{\partial x^{i}} & =0, \quad(i \text { not summed, } H=\log h) .
\end{aligned}
$$

* The completeness of $1+S(\beta+1, \beta, \lambda)$ in $C[0,1]$ is proved for $-1<\beta \leqq 2$ in a paper to appear in the Annals of Mathematics.

$\dagger$ L. P. Eisenhart, Riemannian Geometry, p. 234. 
From (2) we obtain

$$
e_{i} \frac{\partial^{2} \xi^{i}}{\partial x^{j} \partial x^{k}}=-e_{j} \frac{\partial^{2} \xi^{j}}{\partial x^{i} \partial x^{k}}=e_{k} \frac{\partial^{2} \xi^{k}}{\partial x^{i} \partial x^{j}}=-e_{i} \frac{\partial^{2} \xi^{i}}{\partial x^{j} \partial x^{k}}
$$

which gives

$$
(i, j, k \neq \text {, no summing), }
$$

$$
\frac{\partial^{2} \xi^{i}}{\partial x^{j} \partial x^{k}}=0, \quad(i, j, k \neq)
$$

From (3) we have

$$
\frac{\partial \xi^{i}}{\partial x^{i}}=\frac{\partial \xi^{j}}{\partial x^{j}} \equiv \tau, \quad(i, j \text { not summed })
$$

From (2) follow

$$
\begin{aligned}
& e_{i} \frac{\partial^{2} \tau}{\partial x^{j} \partial x^{j}}+e_{j} \frac{\partial^{2} \tau}{\partial x^{2} \partial x^{i}}=0 \\
& e_{j} \frac{\partial^{2} \tau}{\partial x^{k} \partial x^{k}}+e_{k} \frac{\partial^{2} \tau}{\partial x^{j} \partial x^{j}}=0, \\
& e_{k} \frac{\partial^{2} \tau}{\partial x^{i} \partial x^{i}}+e_{i} \frac{\partial^{2} \tau}{\partial x^{k} \partial x^{k}}=0, \quad(i, j, k \text { not summed) }
\end{aligned}
$$

so that $\partial^{2} \tau / \partial x^{i} \partial x^{i}=0$, ( $i$ not summed), and by (4) we get $\partial^{2} \tau / \partial x^{i} \partial x^{j}=0$, so finally we have

$$
\tau=\frac{\partial \xi^{i}}{\partial x^{i}}=a_{0}+a_{j} x^{j}, \quad(i \text { not summed }),
$$

where the $a$ 's are constants. The general solution $\xi^{i}$ of (2) and (5) is found to be

$$
\xi^{i}=b^{i}+a_{0} x^{i}+x^{i} a_{j} x^{j}-\frac{1}{2} a_{i} e_{i} e_{j}\left(x^{j}\right)^{2}+b_{j}{ }^{i} x^{j} .
$$

The $a$ 's and $b$ 's are arbitrary, with $e_{i} b_{j}{ }^{i}+e_{j} b_{i}{ }^{j}=0,(i, j$ not summed). The group generated by the $\xi^{i}$ of $(7)$ is the general conformal group of $(n+1)(n+2) / 2$ parameters. ${ }^{*}$ In order to define a group of motions, the $\xi^{i}$ must satisfy the further conditions (3).

* S. Lie, Theorie der Transformationsgruppen, vol. 3, pp. 334, 347. 
If we substitute the value of $\xi^{i}$ as given by (7) into (3), we tain an equation which can be written as

$$
\sum_{i=1}^{N} A_{i} u^{i}=0, \quad(N=(n+1)(n+2) / 2),
$$

where the $A_{i}$ represent the $N$ constants in the expression for $\xi^{i}$ and $u^{i}$ are functions of the $x$ 's. From (8) we obtain an infinite sequence of equations

$$
\sum_{i=1}^{N} A_{i} \frac{\partial^{t} u^{i}}{\partial x^{a_{1}} \cdots \partial x^{a_{t}}}=0, \quad(t=0,1,2, \cdots),
$$

which must be identically satisfied in the $x$ 's. The function $H$ being assumed analytic in a certain domain of the variables $x$, we may express the $u^{i}$ in the form

$$
u^{i}=u_{0}^{i}+u_{j}^{i} x^{j}+\frac{1}{2 !} u_{j k}^{i} x^{i} x^{k}+\cdots,
$$

and substituting for $u^{i}$ in (9), we see that

$$
\sum_{i=1}^{N} A_{i} u_{a_{1} \cdots a_{t}}^{i}=0, \quad(t=0,1, \cdots) .
$$

Hence a necessary and sufficient condition for the existence of non-zero solutions for $A_{i}$ is that the rank of the matrix

$$
\left\|u_{0}^{i}, u_{j}^{i}, u_{\jmath k}^{i}, \cdots\right\|
$$

be $\leqq N-1$. Since this condition must hold for every point in the domain of analyticity, we can replace the above matrix by

$$
\left\|u^{i}, \frac{\partial u^{i}}{\partial x^{j}}, \frac{\partial^{2} u^{i}}{\partial x^{j} \partial x^{k}}, \cdots\right\| .
$$

This matrix in turn can be replaced by the finite matrix

$$
\left\|u^{i}, \frac{\partial u^{i}}{\partial x^{j}}, \cdots, \frac{\partial^{N-1} u^{i}}{\partial x^{a_{1}} \cdots \partial x^{a} N-1}\right\|,
$$

since we cannot have more than $N$ independent equations (10).*

* Equations of the type (8) have been considered by M.S. Knebelman, who has obtained necessary and sufficient conditions for the existence of constant solutions $A$. 
The $u^{i}$ involve derivatives of $H$ and the condition on the rank of the matrix (11) gives us the required restrictions on $H$. We state this in the following theorem.

Theorem 1. Given a function $H$ defining a conformally fat space, a necessary and sufficient condition that this space admit a group of motions is that the rank of the matrix (11) be $\leqq N-1$, where $N=(n+1)(n+2) / 2$. If the rank is $N-r$, the space admits $a G_{r}$ of motions.

We shall find the conditions for a conformally flat space with $h^{2}=1 / f\left(r^{2}\right)$ to admit a group of motions, where

$$
r^{2}=\sum e_{i}\left(x^{i}\right)^{2}
$$

For this case (3) becomes

$$
\frac{f^{\prime}}{f}=\frac{a_{0}+a_{i} x^{i}}{\sum e_{i} b^{i} x^{i}+\left(2 a_{0}+a_{i} x^{i}\right) r^{2} / 2} .
$$

In order for the right member of the above equation to be a function of $r^{2}$, we must have

$$
a_{i}=b^{i}=0, \quad \text { or } \quad a_{0}=0, \quad e_{i} b^{i}=c a_{i}, \quad(i \text { not summed }) .
$$

For the second case we find that $f\left(r^{2}\right)$ must be of the form

$$
f\left(r^{2}\right)=\left(\alpha r^{2}+\beta\right)^{2}, \quad(\alpha, \beta \text { const. }),
$$

that is, the space is of constant curvature.* For the first case $f$ must be of the form $f\left(r^{2}\right)=\alpha r^{2}$, where $\alpha$ is a constant. This proves the following theorem.

THEOREM 2. The only metric spaces with quadratic form $\left[1 / f\left(r^{2}\right)\right] \sum e_{i}\left(d x^{i}\right)^{2}$ which admit a group of motions are spaces of constant curvature, where $f$ has the form (12), and spaces with the quadraticform $\left(1 / \alpha r^{2}\right) \sum e_{i}\left(d x^{i}\right)^{2}$. The $r^{2}$ has the value $r^{2}=\sum e_{i}\left(x^{i}\right)^{2}$.

It can be shown that if $H=\alpha+\alpha_{i} x^{i}$, the corresponding space admits a group of motions of at least $n-1$ parameters, and if $H=\alpha+(1 / 2) \alpha_{i j} x^{i} x^{j}$, the corresponding space admits a group of motions of at least $n$ parameters, the $\alpha$ 's being arbitrary. This is shown by a consideration of the rank of (11) for these choices of $H$.

* Riemannian Geometry, p. 85. 
3. Simply Transitive Groups. If a motion in $V_{n}$ is to be a translation, we must have $e^{2 H} \sum e_{i}\left(\xi^{i}\right)^{2}=c$, where $c$ is a constant. If we calculate $\partial H / \partial x^{i}$ from this relation and substitute in (3), it is found that $\tau=0$, and hence a necessary condition for a translation is that $\xi^{i}$ be of the form $\xi^{i}=b^{i}+b_{j}^{i} x^{j}$. Suppose $V_{n}$ admits a simply transitive group $G_{n}$ of translations. Then from (3) and the fact that $\tau=0$, it is easily seen that $H$ is constant, that is, the $V_{n}$ is a flat space. This gives us the following theorem.

Theorem 3. If a conformally flat space admits a simply transitive group of translations, the space is flat.

If the $e_{i}$ are all the same sign, it is easily shown that the group must be Abelian.

A simply transitive group $G_{n}$ being given, we consider the conditions under which there exists a conformally flat space admitting $G_{n}$ as a group of motions. Necessary and sufficient conditions are given by

$$
\begin{gathered}
\frac{\partial H}{\partial x^{i}}=L_{i}, \\
e_{i} L_{j k}^{i}+e_{j} L_{i k}^{j}=0, \quad(i, j \text { not summed, } i \neq j),
\end{gathered}
$$

where

$$
\begin{aligned}
L_{j k}^{i}=-\xi_{a \mid k} \frac{\partial \xi_{a \mid}^{i}}{\partial x^{j}}, \quad L_{i}= & L_{1 i}^{1}=\cdots=L_{n i}^{n}, \\
& \left(\xi_{a \mid k} \xi_{b \mid}^{k}=\delta_{b, s}^{a}, \xi_{a \mid i} \xi_{a \mid}^{j}=\delta_{i}^{j}\right) .
\end{aligned}
$$

From (14) and (15) it follows that (13) are completely integrable. Now if $\xi^{i}$ satisfies (14) and (15) we have seen it must be of the form (7), and hence we have the following theorem.

ThEOREM 4. A given simply transitive group will be a group of motions of a conformally flat space if and only if the group is a sub-group of the general conformal group. The determination of the space depends on one arbitrary constant.

North Carolina State College 